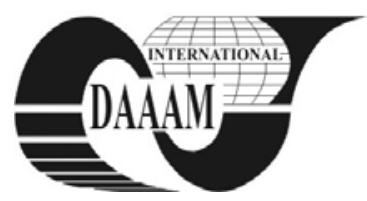

\title{
A LEVENBERG-MARQUARDT METHOD FOR NONLINEAR MODEL PREDICTIVE CONTROL DESIGN
}

\author{
DOKOUPIL, J[akub]; BURLAK, V[ladimir] \& PIVONKA, P[etr]
}

\begin{abstract}
This article deals with a nonlinear model predictive control design (NMPC) which applies numerical optimization using the Levenberg-Marquardt method in iterative batch mode adaptation. The proposed approach enables asymptotic tracking of a reference trajectory by a prediction of a general nonlinear model. Investigation of the properties of the NMPC is performed using the Wiener nonlinear model which is suitable to describe an unknown process dynamics. The work therefore also seeks to formulate the optimal prediction of Wiener model output in state space representation.

Key words: nonlinear model predictive control, Wiener model, Levenberg-Marquardt method, innovative state space model
\end{abstract}

\section{INTRODUCTION}

NMPC falls within a class of controllers optimizing future plant evolution through the use of an explicit mathematical process description. The most widely used approach is linear model predictive control implementation (MPC) (Maeder et al., 2002) applied to a linear or Wiener model quadratic problem (Enso \& Kaddour, 2002). Employing MPC together with Wiener model, which is able to better effect a general nonlinear process, requires a calculation of inversion of static nonlinearity in the feedback loop. Furthermore, given its linear and nonlinear parts, parameterization of the Wiener model might not be unique, which might lead to an incorrect synthesis of MPC.

The aim of this work is to generate the design of a predictive controller directly optimizing the Wiener model prediction. Optimal sequence of action values is newly found based on a LM search direction (Dokoupil \& Pivoňka, 2010). Some works, such as (Ekman, 2008), deal with the design of MPC for bilinear model.

The next area of research will be focused on the design of a predictive controller with a higher order of linear approximation and verification of NMPC using the Hammerstein-Wiener model and its variations.

\section{NONLINEAR MODEL PREDICTIVE CONTROL}

One of a suitable candidate to describe a black-box process behavior is the Wiener model. It consists of a linear dynamic followed by a static nonlinearity. Suppose the Wiener model expressed in a state space representation as follows

$$
\begin{gathered}
x_{k+1}=A x_{k}+B u_{k}+G v_{k} \\
z_{k}=C x_{k}+v_{k} \\
y_{k}=g\left(z_{k}, \eta\right)
\end{gathered}
$$

where $y_{k} \in \mathbb{R}^{l}$ is the measured output, $u_{k} \in \mathbb{R}^{m}$ is the input variable, $z_{k} \in \mathbb{R}^{l}$ is the unmeasurable input to the nonlinear part of model and $x_{k} \in \mathbb{R}^{n}$ is the state of the linear dynamics represented by an innovative state space model. We assume throughout that matrices $(A, B, G, C)$ ensure the reachability and the observability of linear dynamics. The static nonlinearity $g(\cdot, \eta)$ considered here is in a form of polynom of order $p$

$$
g\left(z_{k}, \eta\right)=\sum_{i=0}^{p} \eta_{i} z_{k}^{i}
$$

The unmeasurable variable $v_{k}$ represents disturbing noises and omnipresent modeling imperfections. $v_{k}$ is ideally having the properties of zero mean white noise with known covariance matrix $R_{k}$, ie

$$
\begin{gathered}
v_{k} \sim\left(0, R_{k}\right) \\
E\left\{v_{k} v_{j}^{T}\right\}=R_{k} \delta_{k-j}
\end{gathered}
$$

where $\delta_{k-j}$ is the Kronecker delta function given by

$$
\delta_{k-j}= \begin{cases}1 & i=j \\ 0 & i \neq j\end{cases}
$$

The role of predictive control can be interpreted as a requirement for tracking the reference trajectory by a predicted model output. In order to find the best model prediction which would use quadratic cost function based on a known sequence of input excitation $\left\{u_{k}\right\}$ and the knowledge of the initial state vector $x_{k_{0}}$, the optimal state predictor minimizing the cost function (8) is built first

$$
\hat{x}_{k+i \mid k}=\arg \min _{\hat{x}} E\left\{\left[x_{k+i}-\hat{x}\right]^{T}\left[x_{k+1}-\hat{x}\right]\right\}
$$

which results in

$$
\hat{x}_{k+i \mid k}=A^{i-1}(A-G C) x_{k}+A^{i-1} G y_{k}+\sum_{j=1}^{i} A^{i-j} B u_{k+j-1}(9)
$$

Optimal $i$-step ahead output predictor shall be probably found by solving the following problem

$$
\hat{y}_{k+i \mid k}=\arg \min _{\hat{y}} E\left\{\left[y_{k+i}-\hat{y}\right]^{T}\left[y_{k+i}-\hat{y}\right]\right\}
$$

by substituting for $y_{k+i}$ from the equation (3) and by replacing the vector $x_{k+i}$ by its best possible estimate $\hat{x}_{k+i \mid k}$, we obtain

$$
\hat{y}_{k+i \mid k}=g\left(C \hat{x}_{k+i \mid k}, \eta\right)
$$

rewritten (11) into a compact matrix form results in

$$
\widehat{Y}_{k+N, N}=g\left(\mathcal{O}_{N} x_{k}+\mathcal{K}_{N} y_{k}+\mathcal{T}_{N} U_{k+N-1, N}, \eta\right)
$$

Vectors $\hat{Y}_{k+N, N}$ and $U_{k+N-1, N}$ will henceforth be referred to as vectors of predicted outputs and vectors of inputs. Vector indices will be written following these rules: the first entry of subscript refers to the time index of the vector's top entry and the second entry of subscript refers to the number of predicted values. The matrix $\mathcal{O}_{N}$ will be henceforth referred to as the extended observability matrix, where the subscript corresponds (as in the case of matrices $\mathcal{K}_{N}$ and $\mathcal{T}_{N}$ ) to the number of predicted values. Individual matrices take a form 


$$
\begin{aligned}
& \widehat{Y}_{k+N, N}=\left[\begin{array}{llll}
\hat{y}_{k+N \mid k} & \cdots & \hat{y}_{k+2 \mid k} & \hat{y}_{k+1 \mid k}
\end{array}\right]^{T} \\
& U_{k+N-1, N}=\left[\begin{array}{llll}
u_{k+N-1} & \cdots & u_{k+1} & u_{k}
\end{array}\right]^{T} \\
& \mathcal{O}_{N}=\left[\begin{array}{c}
C A^{N-1}(A-G C) \\
\vdots \\
C A(A-G C) \\
C(A-G C)
\end{array}\right] \quad \mathcal{K}_{N}=\left[\begin{array}{c}
C A^{N-1} G \\
\vdots \\
C A G \\
C G
\end{array}\right] \\
& \mathcal{T}_{N}=\left[\begin{array}{cccc}
C B & \cdots & C A^{N-2} B & C A^{N-1} B \\
& \ddots & \vdots & \vdots \\
& & C B & C A B \\
& & & C B
\end{array}\right]
\end{aligned}
$$

The goal is to minimize the square vector of errors $E_{r, k+N, N, U}$ in prediction horizon $\hat{Y}_{k+N, N}$ of the reference trajectory $W_{k+N, N}$ (Muske \& Badgwell, 2002) which consists of sequences of the desired values

$$
\begin{gathered}
E_{r, k+N, N, U}=W_{k+N, N}-\hat{Y}_{k+N, N} \\
W_{k+N, N}=\left[\begin{array}{llll}
w_{k+N} & \cdots & w_{k+2} & w_{k+1}
\end{array}\right]^{T}
\end{gathered}
$$

and, at the same time, to penalize the invested control energy. As the problem considered here is nonlinear, given the properties of the model, its direct analytical solution is not tractable. Numerical optimization is in this case achieved using the technique of linearization in the neighborhood of $U_{k+N-1, N}^{i-1}$. For the sake of clarity we will employ the following notation $U^{i-1} \leftarrow U_{k+N-1, N}^{i-1}$ and $E_{r, U^{i}} \leftarrow E_{r, k+N, N, U^{i}}$.

For the cost function and its approximation by a first-order Taylor series expressed in a matrix form, we write

$$
\begin{gathered}
V_{N}\left(u_{k+N-1}^{i-1}+\delta u_{k+N-1}^{i}, \cdots, u_{k}^{i-1}+\delta u_{k}^{i}\right) \\
=\left\|E_{r, U^{i}}\right\|_{Q_{e, N}^{2}}^{2}+\left\|U^{i}\right\|_{Q_{u, N}}^{2} \\
\approx\left\|E_{r, U^{i-1}}+\left(\frac{\partial E_{r, i}^{T}{ }^{i-1}}{\partial U^{i-1}}\right)^{T} \delta U^{i}\right\|_{Q_{e, N}}^{2}+\left\|U^{i-1}+\delta U^{i}\right\|_{Q_{u, N}}^{2}
\end{gathered}
$$

in witch $Q_{e, N}$ and $Q_{u, N}$ are positive definite weighting matrices and with the requirement of distance reduction $\delta U^{i}=U^{i}-$ $U^{i-1}$. A numerically more attractive approach, which uses the Levenberg-Marquardt techniques, will be achieved by expansion of the cost function (19) by adding a restrictive condition which will require minimal variation in $\delta U^{i}$. By formulating a multicriterial problem and applying the Lagrange multiplier $\lambda$ which works with a set of positive real numbers, we obtain

$$
\begin{aligned}
& V_{N, \lambda}\left(u_{k+N-1}^{i-1}+\delta u_{k+N-1}^{i}, \cdots, u_{k}^{i-1}+\delta u_{k}^{i}\right) \\
& \quad=V_{N}\left(u_{k+N-1}^{i-1}+\delta u_{k+N-1}^{i}, \cdots, u_{k}^{i-1}+\delta u_{k}^{i}\right)+\lambda\left\|\delta U^{i}\right\|_{2}^{2}
\end{aligned}
$$

The linear problem of least-squares is solved in relation to the operation of $\delta U^{i}$ in the linear approximation of the cost function $V_{N, \lambda}$

$$
\left\{\delta u_{k+N-1}^{* i}, \cdots, \delta u_{k}^{* i}\right\}=\arg \min _{\delta u_{k+N-1}^{i}, \cdots, \delta u_{k}^{i}} V_{N, \lambda}
$$

and as a result we obtain a relation for iterative actualization of sequence of control actions

$$
\begin{gathered}
U^{i}=\left[\Psi_{r, U^{i-1}}^{T} Q_{e, N} \Psi_{r, U^{i-1}}+Q_{u, N}+\lambda I\right]^{-1} \\
\times\left[\Psi_{r, U^{i-1}}^{T} Q_{e, N}\left(E_{r, U^{i-1}}+\Psi_{r, U^{i-1}} U^{i-1}\right)+\lambda U^{i-1}\right]
\end{gathered}
$$

where $\Psi_{r, U^{i-1}}=\left[\frac{\partial \hat{Y}_{k+N, N}^{T}}{\partial U^{i-1}}\right]^{T}$.

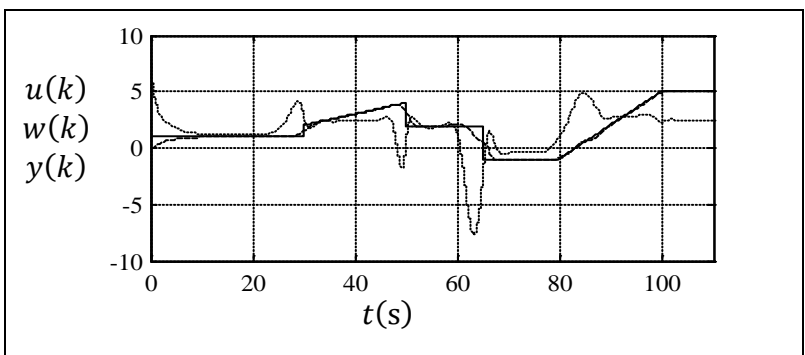

Fig. 1. Direct application of NMPC to the Wiener process control. Dashed line $u(k)$, solid line $w(k)$, dot-dashed line $y(k)$.

\section{SIMULATION EXAMPLE}

In this section, NMPC practical properties are examined via simulation example. The Wiener system to be controlled has static nonlinearity given by $g\left(z_{k}, \eta\right)=z_{k}-0.5 z_{k}^{2}+0.1 z_{k}^{3}$ and its corresponding linear part by $\frac{y(s)}{u(s)}=\frac{2}{(10 s+1)(s+1)^{2}}$. The result of the Wiener process control by suggested NMPC is shown in Fig. 1., where $\lambda=0.01, N=100, Q_{e, N}=I, Q_{u, N}=$ $0.015 I$.

\section{CONCLUSION}

This paper presents and develops the strategy of NMPC design which is based on a LM search direction. The simulations result shows that the proposed approach is effective in the tracking capability of referencing trajectory of the Wiener model without the need to calculate the inversion of static nonlinearity. It should be mentioned, that the theoretical aspects of algorithm provides its implementation in the cases, when other types of nonlinear model structures (as Hammerstein-Wiener etc.) are introduced.

\section{ACKNOWLEDGEMENTS}

This work was supported by grant „Research of Modern Methods and Approaches in Automation“ from the Internal Grant Agency of Brno University of Technology (grant No. FEKT-S-11-6) and by grant „Mobile Sensoric Systems and Networks“ from the Grant Agency of the Czech Republic (102/09/H081 SYNERGY).

\section{REFERENCES}

Dokoupil, J.; Pivoňka, P. (2010). A Levenberg-Marquardt Algorithm with Instrumental Variables and its Application on the Identification of Dynamic System, Annals of $D A A A M$ for 2010 \& Proceedings of the 21st International DAAAM Symposium, 20-23rd October 2010, Zadar, Croatia, ISSN 1726-9679, ISBN 978-3-901509-73-5, Katalinic, B. (Ed.), pp. 1359-1360, DAAAM International Vienna, Vienna

Ekman, M. (2008). Bilinear Black-Box Identification and MPC of the Activated Sludge Process. Journal of Process Control, Vol. 18, No. 7, 643-653, ISSN 0959-1524

Enso, I.; Kaddour, N. (2002). Advanced Process Identification, Marcel Dekker, Inc., ISBN 0-8247-0648-X, New York

Maeder, U.; Borrelli, F. \& Morari, M. (2002). Linear OffsetFree Model Predictive Control. Automatica, Vol. 42, No. 10, 2214-2222, ISSN 0005-1098

Muske, K.; Badgwell, T. (2002). Disturbance Modeling for Offset-Free Linear Model Predictive Control. Journal of Process Control, Vol. 12, No. 5, 617-632, ISSN 0959-1524 